
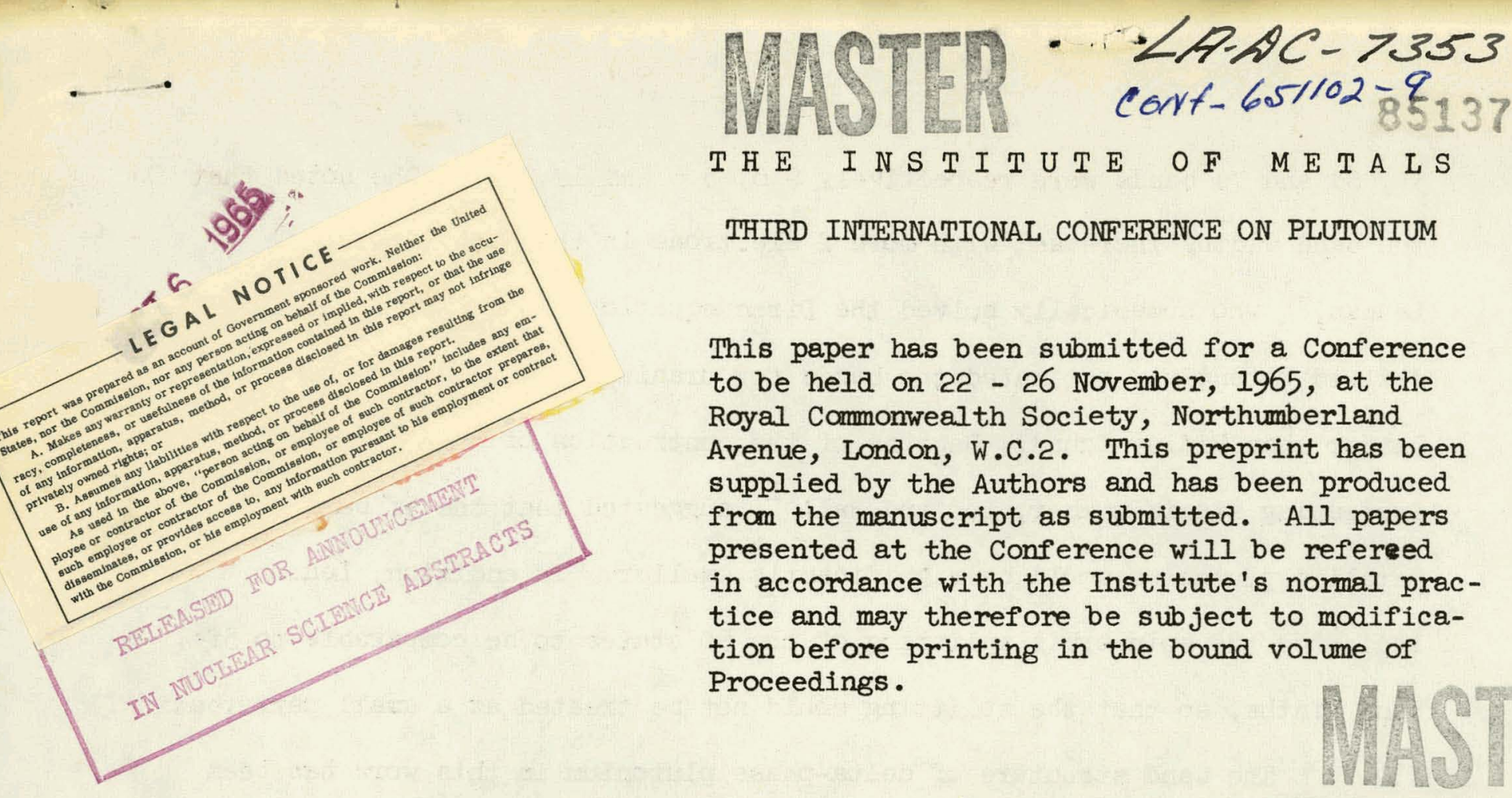

THE INSTITUTE OF METALS

THIRD INTERNATIONAL CONFERENCE ON PLUTONIUM

This paper has been submitted for a Conference to be held on $22-26$ November, 1965, at the Royal Commonwealth Society, Northumberland Avenue, London, W.C.2. This preprint has been supplied by the Authors and has been produced from the manuscript as submitted. All papers presented at the Conference will be refereed in accordance with the Institute's normal practice and may therefore be subject to modification before printing in the bound volume of Proceedings.

\title{
ELECTRONIC BAND STRUCTURES OF PLUTONIUM AND ITS MONOCARBIDE
}

By

E. A. Kmetko, J. T. Waber

(Ios Alamos Scientific Laboratory)

The band structure of delta-phase plutonium has been calculated by the tight binding and the Augmented Plane Wave methods. The band width, density of states curve and the Fermi surface were obtained from these calculations. The width of the $5 \mathrm{f}$ bands from the tight binding results is in better agreement with the width Kmetko deduced from a heuristic study than with that deduced from the Augmented Plane Wave results. The width of the $5 f$ band in PuC was found by the tight binding method to be about two-thirds as large as that in delta-phase plutonium.

\section{Introduction}

1.1 The first important estimate of the band width of plutonium was provided by Lee and Ha.l. ${ }^{(1)}$ and based on Ridley's ${ }^{(2)}$ results. She calculated the self-consistent field (SCF) eigenvalues for a uranium atom confined to a Wigner-Seitz sphere, the radius of which was appropriate to the B.C.C. gamma-uranium phase. By using another boundary condition (that wave functions vanish at the cell boundary) she obtained exaggerated energy ranges for the various states. By comparing similarly derived results for copper to those obtained by Howarth ${ }^{(3)}$ using the cellular method, she estimated that the bands would be one-third as wide as her corresponding energy ranges. For the configuration $\left(5 f^{2} 6 d^{2} 7 s^{2}\right)$ of uranium, her estimated band widths of the 


\section{DISCLAIMER}

This report was prepared as an account of work sponsored by an agency of the United States Government. Neither the United States Government nor any agency Thereof, nor any of their employees, makes any warranty, express or implied, or assumes any legal liability or responsibility for the accuracy, completeness, or usefulness of any information, apparatus, product, or process disclosed, or represents that its use would not infringe privately owned rights. Reference herein to any specific commercial product, process, or service by trade name, trademark, manufacturer, or otherwise does not necessarily constitute or imply its endorsement, recommendation, or favoring by the United States Government or any agency thereof. The views and opinions of authors expressed herein do not necessarily state or reflect those of the United States Government or any agency thereof. 


\section{DISCLAIMER}

Portions of this document may be illegible in electronic image products. Images are produced from the best available original document. 
$5 f, 6 \mathrm{~d}$ and $7 \mathrm{~s}$ bands were respectively $0.6,5.6$ and $12.9 \mathrm{eV}$. She noted that the band widths increased with more $f$ electrons in the configuration. Lehman, ${ }^{(4)}$ who numerically solved the Dirac equation using a statistically derived potential, estimated the bands for uranium to be about 20 per cent larger than Ridley found. Because of the contraction of the $5 f$ shells with increasing atomic number, Iee and Hall ${ }^{(I)}$ suggested that the $5 f$ band width in metallic plutonium would be significantly smaller. In addition, Lehman indicated the spin-orbit splitting of the $5 \mathrm{f}$ states to be comparable to $5 f$ band widths, so that the splitting could not be treated as a small perturbation. 1.2. The band structure of delta-phase plutonium in this work has been investigated from two different standpoints. Not only were different methods of calculation employed but the wave functions and the crystal potentials were also different. One of these is the Augmented Plane Wave (APW) method of Slater, (5) which involves solution of the Schroedinger equation for the crystal potential $V(r)$. The second is the tight binding (TB) method ${ }^{(6)}$ which treats the difference between the free atom and the crystal potential as a small perturbation. Both methods are non-relativistic.

1.3. Initially the goal was simply to obtain the band width from theoretical calculations rather than inferentially. Kmetko( ${ }^{(7)}$ had been able to explain the high electronic specific heat, the paramagnetism and the negative coefficient of delta-phase plutonium by using two rigid bands as a heuristic model, provided the width of the $5 f$ bands was narrow enough. The TB method was a logical method of obtaining the band width. Subsequently, it appeared possible to obtain this and further information about the Fermi surface and the density of states curves from an APW calculation.

1.4. To gain further insight into the behavior of plutonium, and the effect of foreign atoms, two additional $T B$ calculations were made. The $5 f$ bands were obtained for PuC. Since the metal sublattice is F.C.C. in this compound and the spacing is about 7 per cent larger than in the delta phase, the $5 \mathrm{f}$ bands 
were also obtained for an expanded plutonium F.C.C. lattlce.

1.5. The present study of PuC is very similar to the TB calculation by Costa and Conte ${ }^{(8)}$ for TiC. Erm and Switendick, ${ }^{(9)}$ using the APW method, have made a more complete study of the band structures of TiC, TiO and TiN. Since few calculations of energy bands have been made by both methods, some evaluation of their relative effectiveness can be made by a study of the two sets of results.

1.6. The present TB calculation yields a band width which is in good agreement with the width of the $5 f$ bands that Kmetko ${ }^{(4)}$ needed to explain the physical properties of delta plutonium. In contrast, the APW calculations yield a much larger band width and a lower density of states $N\left(E_{F}\right)$ at the Fermi level $\mathrm{F}_{\mathrm{F}}$.

\section{Tight Binding Calculation - Description of Technique}

2.1. The non-relativistic TB method ${ }^{(6)}$ was employed to obtain an estimate of the $5 f$ band widths in (a) delta plutonium; (b) the metal lattice as it exists in PuC, i.e., expanded delta plutonium; and (c) PuC itself, which has the NaCl structure. Interactions with other bands were not considered. 2.2. The atoms in PuC were assumed to be neutral for the sake of simplicity as. well as because the three $2 p$ orbitals in carbon comprise a natural set to form covalent bonds with the neighboring plutonium atoms. (10) The wave functions used were the non-relativistic Hartree-Fock-Slater type; (II) for plutoniun only the functions for: the $(5 f)^{6}(7 s)^{2}$ configuration were available in the tabulation of Herman and Skillman. (11) It seems likely that configurations involving the $6 \mathrm{~d}$ electrons would be more realistic. It is felt, however, that the principal goal of this study, the rough estimate of the $5 f$ band width, could be achieved notwithstanding this deficiency. 2.3. The one-electron crystal potential $V(r)$ for the metal lattice was constructed from the potential of the neutral atom that had been subjected to the appropriate Wigner-Seitz boundary condition. (12) Thus the requirement 
that each cell, the central one excepted, be neutral is automatically satisfied. The potentials were obtained from a computer program developed by Barnes (13) for a quantum- and correlation-corrected Thomas-Fermi-Dirac equation. For the atom situated in the central sphere the problem of the potential requires more scmatiny. The potential computed for a singly ionized atom subject to the proper Wigner-Seitz boundary will not suffice inasmuch as the radius of a free ion is very slightly larger than the radius of the Wigner-Seitz sphere. The neutral atcon should first be squeezed to $s$ ize after which an electron is removed (to take account of self interaction) while the remaining charge distribution is unaltered. A rather simple gpproximation to such a potential may be obtained by adding a coulombic term $(-l / r)$ to the potential of a suitably squeezed neptunium atom. Inasmuch as the potentials for neptunium and plutonium differ by less than 1 per cent at all radii, it makes little difference which potential is actually used in the central cell. This is responsible for a minor simplification in programing but cannot be applied for much lighter atoms since the relative change in nuclear charge is greater.

2.4. The crystal potential for the PuC lattice was obtained by adding the potential of the neutral free carbon atom at each site in the neighborhood. 2.5. In the TB method one is concerned with the two-center integrals *

$$
\int \phi_{i}(r)[V(r)-U(r)] \phi_{j}\left(r-R_{l}\right) d v
$$

where $U(r)$ is the potential of a free atom at the origin and $\phi_{i}(r)$ and $\phi_{j}\left(r-R_{l}\right)$ are the $i$ th and $j$ th atomic wave-functions situated at the origin and position $\mathrm{R}_{\ell}$, respectively. The coulombic terms cancel so that only the potentials for the neutral atom need be taken.

* Subsequent integrals in this paper will be abbreviated to the form $<\phi_{i}(r)|F| \phi_{j}\left(r-R_{\ell}\right)>$ where $F$ is an appropriate function or operator. 
2.6. The integrals were obtained in a direct manner. The volume about the atoms is divided into cubes. At the center of each cube the wave functions and potentials are evaluated by tabular interpolation except for the angular factors which are supplied by a subprogram involving the analytic expressions. The mesh size as well as the extent of the volume in the three coordinate directions is arbitrary and the values are dictated solely by the compromise necessary between coarseness of the result and available computer time. A mesh defined by having a cube 51 points to an edge and centered about each plutonium atom was found to be adequate.

2.7. The program is written so that matrix elements can be computed between any pairs of atoms in the simple cubic, F.C.C., B.C.C., NaCl and CsC crystal types so that farther neighbors than the first may be conveniently considered.

2.8. Overlap integrals $\left\langle\phi_{i}(r)\right| \phi_{j}\left(r-R_{l}\right)>$ and self-energy integrals $<\phi_{i}(r)|V(r)-U(r)| \phi_{i}(r)>$ as well as two-center integrals may be computed merely by supplying the program with the appropriate index number. The criterion for the validity of the TB method is that the overlap integrals must be much smaller than $1 .(14)$ In the present case, they were all less than $10^{-2}$; the contributions from the second nearest neighbors were of the order of $10^{-4}$ and thus could be ignored. The s-f interactions, which are not included, were found to be of the order of $10^{-2}$. If a binary lattice is being considered, the potential of the second type of atom is added automatically by the program to that of the metal lattice.

2.9. The energy eigenvalues $E$, as they depend upon the wave vector $\vec{k}$, were obtained by a separate program which finds the roots of the secular equation $\left|H_{i j}-\left(C_{i}-E_{i}^{0}-E\right) \delta_{i j}\right|=0$, where the $H_{i j}$ are the matrix elements in Eq. (1), $C_{i}$ are the self energy terms and $E_{i}^{O}$ are the eigenvalues corresponding to the ith state of the free atom.

3. Results and Discussion of Tight Binding Calculations

3.1 Curves showing the dependence of $\mathrm{E}$ vs $\mathrm{k}$ along the (100) direction, 
i.e. $\Gamma-\Delta-X$; are shown in Figs. 1,2 and 3 for delta plutonium, the deltaplutonium lattice expanded to the size found in PuC, and PuC itself, respectively. "The notation follows that of Bouckaert, Smoluchowski and Wigner (15) except that the primed states are marked with an asterisk to show them more clearly. The general character of the delta-plutonium bands is preserved as the lattice expands. The inflection points and maxima seem to undergo little if any shift; only the width changes. A linear expansion of the lattice by only $\sim 7$ per cent causes the band width to decrease to approximately half of its original value. In Fig. 3, the perturbation of the bands by the carbon atom potential is quite evident as a slight rearrangement of levels as well as an overall broadening of the bands. The individual states are perturbed differently than in the pure metal lattice, as discussed below, since the nearest carbon atoms are situated in an octahedral configuration. 3.2. The overall width of the $5 \mathrm{f}$ band in delta plutonium is close to $1 \mathrm{eV}$. However, the band obviously has a rather high peak, $0.77 \mathrm{eV}$ wide, in which the average density of states is about 20 (spin degeneracy included). 3.3 In an earlier investigation, ${ }^{(7)}$ a two-band model was applied to delta plutonium.' General agreement between model and experiment was obtained for a $\left(5 f \frac{5}{2}\right)$ band of width between $\sim 0.25$ and $\sim 0.50 \mathrm{eV}$ superimposed upon a broad band having an average density of state of about 4 states/(atom-eV) in the vicinity of the $\left(5 f \frac{5}{2}\right)$ band. There is obviously a general agreement, therefore, between the results of this probably oversimplified IB calculation and the previous result.

3.4 The most strongly perturbed $f$ states are those in the triply degenerate subset designated as $f_{\epsilon}$, which have angular dependence of the type $x\left(y^{2}-z^{2}\right) / r^{3}$. They are the only ones having anti-nodes in the directions of the nearest neighboring metal atoms. The $f_{\epsilon}$ functions give rise to the rather strongly $k$ dependent states along the curves $r_{25}^{\prime}-\Delta_{2}-x_{7}^{*}$ and $\boldsymbol{r}_{25}^{\prime}-\Delta_{5}-x_{5}^{*}$ shown in Figs. 1,2 and 3 . Of the other four functions, the 
singly degenerate $f_{\beta}$ with $x y z / r^{3}$ dependence has nodar planes in line with all of the nearest metal atoms, but anti-nodes face the sites of the second nearest carbon atcms. For the remaining triply degenerate states $f_{\delta}$ of type $x\left(5 x^{2}-r^{2}\right) / r^{3}$, the nearest metal atoms lie on nodal planes and in the vicinity of nodal cones; the nearest carbons, however, lie directly along the anti-nodal lines. With the pertinent geometry of the $f$ functions thus in mind, the dependence of each of the various states upon the wave vector can be more readily understood. As a particular example, notice how the energy of the $\Gamma_{2}^{*}$. state is depressed in changing from the expanded metal to the carbide, because of the inclusion of the carbon potential in the self-energy term, while the $k$-dependence is increased as is evidenced by the rise in the energy $\mathrm{x}_{3}$

3.5. It is apparent that the metal-carbon atom interactions can be quite strong. These will be taken into account in further work. It is also planned to include the $5 f-7 s, 5 f-6 d$ interactions, as these could have a profound effect upon the calculated bands.

4. Augmented Plane Wave Method - Description of Calculation 4.1. The non-relativistic APW method was first proposed by Slater (5) in 1937. The principal computational details of this method were presented by Wood ${ }^{(16)}$ in 1961 . In terms of convergence of the series of terms, two parameters are important, nomely the angular momentum $\ell$ and the magnitude $\mathrm{k}_{\max }$ of the largest vector included in the set. On the basis of the behavior of the spherical Bessel functions $j_{l}\left(\frac{\pi k r}{a}\right)$, which are solutions in the muffin tin or constant potential region, $\& \geq 2 k_{\max }$ to insure convergence. Typical roots at $x_{3}^{*}$ are 0.769959 for $\ell=9,0.787859$ for $\ell=12$ and 0.787827 for $\ell=13$, the value of $k_{\max }$ having been chosen as 6 .

4.2. The crystal potential was constructed by the method outlined by Mattheiss. (17) More specifically, the relativistic Dirac-Slater wave functions werc used to compute the charge densities, and the charge density from a neighboring atom, which overlapped the charge density $\rho$ of the central atom, 
was obtained by Löwdin's alpha expansion. (18) These contributions out to and including the fourth nearest neighbors were sumed and spherically averaged. The exchange potential was obtained from the total spherically averaged charge density by Slater's free electron or $p^{1 / 3}$ method. (19) For plutonium in the $f^{6} s^{2}$ configuration, the relativistic wave functions appropriate to the free atom were used. However, for the other configuration, $f^{4.75} d^{1.25} s^{2}$, the relativistic wave functions for the atom confined to the -. delta-phase Wigner-Seitz sphere were used to compute the charge density $p$. Here, overlap from other than the set of first nearest neighbors is unimportant. Use of the confined atomic wave functions and the appropriate potential brings the calculation for this configuration closer to the $\mathbb{T}$ calculation, in which the confined potential was also used. For both conf'igurations, the APW radius $R_{s}$ was chosen to equal the metallic radius, or half of the contact distance. In the present atomic units, the value used was 3.09 Bohr or $1.642 \AA$.

4.3. The mesh used in the Brillouin zone can be represented by $\left(\frac{m}{2}, \frac{n}{2}, \frac{p}{2}\right)$ and corresponds to 256 points. In certain directions, such as from $\Gamma$ to $L$, solutions were obtained at the points $\left(\frac{n}{8}, \frac{n}{8}, \frac{n}{8}\right)$. Such points were used in drawing the $E(k)$ curves. However, they were not used in constructing the density of states curve.

\section{Band Structure and Density of States Curves}

5.1. The band structure or $\mathrm{E}(\mathrm{k})$ curves for one of the high symmetry directions are presented in Fig. 4 for plutonium in two configurations, namely $f^{6} s^{2}$ and $f^{4.75} d^{1.25} s^{2}$. In both cases, the band derived principaliy from $7 \mathrm{~s}$ states lies above the $d$ bands. This situation is in contrast to that in the lighter transition metals. The muffin tin potentials for the two configurations are presented in this graph.

5.2. It will be seen that the bands for the configuration $f^{4.75} \mathrm{~d}^{1.25} \mathrm{~s}^{2}$ are very similar to those found for $f^{6} s^{2}$. The most noticeable feature. is the sight relocation of the various bands. Because the $f$ states have lower 
eigenvalues in the $f^{4.75} \mathrm{~d}^{1.25} \mathrm{~s}^{2}$ configuration, there is somewhat less interaction with the $d$ bands. For this reason, the $E(k)$ curves nearly become monotonic functions of the wave vector $\vec{k}$. The $6 s$ and $6 \mathrm{p}$ bands are nearer the 6d bands. These general trends are supported by the atomic one-electron eigenvalues. However, the variation with $x$ would appear to be smaller for the band calculation than for the atomic calculation.

5.3. A more complete set of $\mathrm{E}(\mathrm{k})$ curves for the various directions within the Brillouin zone and on its surface is presented in Fig. 5. These curves illustrate the complex relations between bands and the decided departure from the bands expected for free electrons.

5.4. Density of states curves $\mathrm{N}(\mathrm{E})$ were prepared for plutonium in the $f^{6} s^{2}$ configuration. Because of the relative similarity between the two APW calculations that differed only in assumed configuration, the density of states curve has not been calculated for $f^{4 \cdot 75} \mathrm{~d}^{1.25} \mathrm{~s}^{2}$. In Fig. 6 the AFW bar graph representing $N(E)$ is shown for $\Delta E=1 / 80$ Rydberg, which is equal to the width of a bar of the histogram. The value of $N(E)$, or more accurately $\Delta \mathrm{N} / \Delta \mathrm{E}$, in any energy region is dependent on two factors. The first is the total number of points in the Brillouin zone at which solutions were obtained, i.e. which were "sampled", and the second is the energy interval. Burdick (20) described a procedure for obtaining a "stable" density of states curve with 2048 points in the Brillouin zone. However, this time consuming process was not used. In general, the smaller the interval $\Delta \mathrm{E}$ used, the more "fine structure" appears. It is thought that because of the narrow dense $f$ bands of plutonium, the $\mathbb{N}(E)$ graph resulting from using $\Delta E=1 / 80$ is not specious. 5.5. The $N(E)$ curve derived from the roots also obtained at the points $\left(\frac{n}{2}, \frac{m}{2}, \frac{p}{2}\right)$ by the $T B$ method are shown in Fig. 7 . No attempt was made to locate the Fermi level, because of its dependence on the positions of the remaining bands. Here the maximum density of states is about 3 times larger than that found by the APW method. To facilitate comparison, the same interval, 1/80 
Rydberg $(0.17 \mathrm{eV})$, was also used for this figure. The region near the center of the band with a density of states over 20.7 states/(eV-atom), is consistent with the numbers Kmetko( ${ }^{(7)}$ deduced from his heuristic study of the properties of plutonium.

5.6. The energy contour for the fourth band holding the seventh and eighth electrons is shown in Fig. 8. Within the confines of this calculation, this energy contour can be regarded as a Fermi surface. This should not be taken seriously in terms of predicting experimental results since the important but neglected relativistic effects will lead to a substantial modification of the bands and consequently of the Fermi surface. The surface drawn is specifically for the APW calculation. However, the shepe would not be expected to be much different for the fourth band obtained using the assumed configuration $f^{4.75} \mathrm{~d}^{1.25} \mathrm{~s}^{2}$ with the AFW program or from the TB calculation. In either calculation, the third band would have a much different appearance. The surface is complex. " Columns extend towards each of the four W. corners of the six square faces.

\section{Relevant Atomic Calculations}

6.1. In order to provide an insight and a guide for evaluating the present work, it is desirable to review the results of atomic and cellular calculations on plutonium as well as other work. A substantial variation of the band locations in cerium, depending on the electronic configuration that was assumed in generating the crystal potential, was recently found in an APW calculation by Wober and Switendick. (2l) A similar but less drastic effect was found for the band structure of nickel by Snow, Waber and Switendick. (22) on the basis of atomic calculations discussed below, it is reasonable to study plutonium in two configurations, namely the ground state $\left(5 f^{5}\right)^{6}\left(7 \mathrm{~s} \frac{1}{2}\right)^{2}$ and one such as $\left(5 \mathrm{f} \frac{5}{2}\right)^{4.75}\left(6 \mathrm{~d} \frac{3}{2}\right)^{1.25}\left(7 \mathrm{~s} \frac{1}{2}\right)^{2}$. From these calculations, one can conclude that the effect of configuration is not large. Furthermore, since a non-relativistic band calculation has been made, and the 
effect of spin-orbital splitting, Darwin and mass-velocity corrections will result in a more complex band structure, further investigation of the effect of configuration would be pointless at this time.

6.2. The general effect of configuration on the bands in cerium was first revealed in atomic (SCF Hartree) calculations by Larson and Waber (23) for free atoms and subsequently by Waber, Iiberman and Cromer ${ }^{(24)}$ for atoms confined within a Wigner-Seitz sphere. It is desirable to review the results of atamic and cellular SCF calculations for plutonium before discussing the electronic band structure further.

6.3. The one-electron eigenvalues for the free atom have been obtained by several methods. Boyd, Larson and Waber ${ }^{(25)}$ used the simple Hartree model for several configurations. Herman and Skillman (1l) studied only the $f^{6} s^{2}$ configuration by the Hartree-Fock-Slater method. The relativistic DiracSlater method discussed by Liberman, Waber and Cromer ${ }^{(26)}$ was used for three configurations. The eigenvalues for the outer electrons are compared in Table I; several trends are indicated. The effect of exchange can be seen by comparing the Hartree values (without exchange) with the Hartree-FockSlater values (with Slater's $\rho^{1 / 3}$ exchange) for the $f^{6} s^{2}$ configuration. The electrons with high angular momenta, and hence many common electrons in the same atomic subshell, are bound more tightly by the inclusion of the exchange potential. The electrons having low angular momenta are not greatly affected. Some of the effects of including the relativistic terms can be seen by comparing the Hartree and the Dirac-Slater eigenvalues. The direct relativistic effect (that arises from the mass-velocity and Darwin terms) is to bind electrons of low angular momenta more tightly. These electrons then shield the nuclear charge more effectively and thus the $5 f$ electrons, in particular, have more positive eigenvalues (smaller numerical values in Table I). This is the indirect relativistic effect discussed by Boyd, Larson and Waber. (27) The third uniform trend is that all of the one-electron eigenvalues (listed 
in Table I) become more negative as the $x$ number of d electrons increases in the general configuration $f^{6-x} \cdot \mathrm{d}^{\mathrm{x}} \mathrm{s}^{2}$. The latter trend is also present in the results of the cellular calculations described below.

6.4. The one-electron energy eigenvalues for a plutonium atom confined to the Wigner-Seitz sphere appropriate to delta plutonium, namely $R_{w s}=3.46$ Bohr units, are plotted in Fig. 9. The ground state normally assumed for the free atom is $\left(7 \mathrm{~s} \frac{7}{2}\right)^{2}\left(5 \mathrm{f}^{\frac{5}{2}}\right)^{6}$. This configuration would be "acceptable" in the metallic solid since it is consistent with the observed lack of a residual magnetic moment on the atom and only a weak Pauli spin paramagnetism. (28) That is, the six $5 f$ electrons complete the $j=5 / 2$ subshell and are so arranged as to have a zero net $M_{j}$ camponent, $i . e .$, no magnetic moment. This configuration is also consistent with delta plutonium having a close-packed structure, which strongly implies a spherical charge distribution.

6.5. Two other general configurations are considered in Fig. 9. In the first, electrons are transferred from the $j=5 / 2$ to the $j=7 / 2$ subshell -the energy separation between these arises from the spin-orbit splitting. The notation for the general configuration $\left(5 f \frac{5}{2}\right)^{6-x}\left(5 f \frac{7}{2}\right)^{x}\left(7 \mathrm{~s} \frac{1}{2}\right)^{2}$ is abbreviated to $f^{6-x_{f} x_{s}{ }^{2}}$. The other alternative involves the transfer from the $\left(5 f \frac{5}{2}\right)$ subshell to the $\left(6 \mathrm{~d} \frac{3}{2}\right)$ subshell and is designated $f^{6-x_{1} x_{s}{ }^{2}}$. The eigenvalue becomes more negative, i.e., the electrons become more tightly bound, as $x$ increases in the configuration $f^{6-x_{d}} x^{2}$. This graph suggests that the configuration $f^{4} d^{2} s^{2}$ would be the most stable one considered. This deduction is based only on a few of the one-electron energies.

6.6. The energy eigenvalues obtained by Herman and Skillman (11) are also plotted In Fig. 9. The order is opposite to the Dirac-slater results, that is, the HFS energy of the $7 \mathrm{~s}$ electron is more positive than that of the $5 \mathrm{f}$ electron. The order of Dirac-Slater results probably arlses from the indirect relativistic effect. The relativistic mass-velocity and other tems which are not included in the band structure calculation, may be one reason why the 
order of the energies of valence electrons is reversed.

6.7. A further reason for the reversal comes from the modification of the potential when the atom is confined to the Wigner-Seitz sphere. Use of the confined atomic functions in the APW calculation for $f^{4.75} \mathrm{~d}^{1.25} \mathrm{~s}^{2}$ did not produce large shifts or significant rearrangement of the $E(k)$ curves. Thus one may surmise that most of the cause for the reversed order in Fig. 9 arises from the indirect relativistic effect.

6.8. The total energy (average of the configuration) was computed by the method of Snow, Canfield and Waber; (29) i.e., the eigenvalues were summed and the total contributions from the two-electron direct and exchange potentials were included. The total energy $E_{T}$ curves are shown in Fig. 10 for the two general configurations discussed above. Three sizes of the Wigner-Seitz sphere are presented, namely $R_{w s}=3.46$ Bohr corresponding to the density of the delta phase, 3.20 Bohr corresponding to the density of the alpha phase and 60.0 Bohr corresponding to the free atom. The net effect of transferring an electron to the $\left(5 \mathrm{f} \frac{7}{2}\right)$ subshell is relatively small despite the significant spin-orbit coupling. The net effect of transferring an electron to the $\left(6 \mathrm{~d} \frac{3}{2}\right)$ subshell is more favorable. It will be seen that the most stable configuration depends upon the density of atoms in the solid. A third general configuration $\left(5 f^{5}\right)^{6}\left(6 d \frac{3}{2}\right)^{y}\left(7 s \frac{1}{2}\right)^{2-y}$, abbreviated as $f^{6} d^{y} s^{2-y}$, has been included for the deltaphase density. Cenerally it is found in the band structure calculations of transition metals that the states occupied in the band derived from $4 \mathrm{~s}$ atomic states contain less than 2 electrons, despite the fact that the ground state configuration of the atom can be represented by $4 s^{2} 3 d^{n}$. In the case of. delta plutonium, Fig. 10 indicates that it would cost energy to transfer electrons from.7s to $6 \mathrm{~d}$ states.

6.9. A complementary plot illustrating the variation of total energy with configuration is shown in Fig. 11. The most stable configuration of the nearly free atom (large sphere radius $R_{\max }$ ) is $f^{6} s^{2}$. At the radius corresponding 
to the delta phase it is $f^{5} \mathrm{ds}^{2}$, and on further compression the $\mathrm{f}^{4} \mathrm{~d}^{2} \mathrm{~s}^{2}$ configuration is favored. The heat of vaporization estimated from this graph is about 60 kcal.

7. Discussion

7.1. The $f$ bands found by the TB method lie near the $5 f$ eigenvalue found by Herman and Skiliman. This value is more negative than that found by the Dirac-Slater method. Consequently, the Herman-Skillmain $5 f$ wave function describes the electrons as being more tightly bound than does the corresponding Dirac-Slater wave function. This may account, in part, for the discrepancy between the widths of the $5 f$ bands as found by the two methods. Generally the $1 B$ method does seem to provide narrower bands than do other methods. Although the $7 \mathrm{~s}$ band was not computed, possibly it would overlap the $5 f$ band because of the large anticipated breadth as well as the significant downward shift of the entire band caused by the large self-energy term $\left.<\phi_{7 \mathrm{~s}}(\vec{r})|V-U| \phi_{7 \mathrm{~s}}(\vec{r})\right\rangle$. The large overlap of $7 \mathrm{~s}$ function on neighboring atoms violates the validity criterion of the TB method $(14)$ so that little would actually be gained by computing the $7 \mathrm{~s}$ band. The separation between $7 \mathrm{~s}$ and $5 f$ states in the free atom is approximately 0.7 Kydberg. If the self energy and broadening is not large enough to cause substantial band overlap, then only the low lying $f$ bands, which can acconmodate 14 electrons, would be occupied. Since 8 electrons are available outside the radon core, the $7 \mathrm{~s}$ bands would not be occupied in this case. Without the inclusion of $7 \mathrm{~s}-5 \mathrm{f}$ interactions into the matrix, it is difficult to be sure whether sufficient overlap does occur.

7.2. The $\left(5 f \frac{5}{2}\right)$ and $\left(5 f \frac{7}{2}\right)$ states of the confined or free atom are separated by $\sim 0.1$ Rydberg as Fig. 9 shows. If the perturbations are actually as weak as implied by the TB calculations, the $f$ bands would almost be split into two separate sub-bands. In the absence of an overlapping $6 \mathrm{~d}$ band, such a large splitting would provide a completely filled $\left(5 \mathrm{f} \frac{5}{2}\right)$ sub-band and two electrons 
would spill over into the higher $j$ sub-band. Within the framework of Kmetko's two-band model, the interpretation of the negative coefficient of expansion of delta plutonium as being due to a decrease in $5 f$ band population with increasing temperature $\left(30^{\circ}\right)(7)$ requires the $\left(5 f \frac{5}{2}\right)$ sub-band to be at least somewhat more than half occupied. With this requirement met, the Fermi level will rise rapidly with increasing temperature. Therefore the necessary condition is that the $6 \mathrm{~d}$ band overlap the lower $5 f$ sub-band.

7.3. Unfortunately, $6 \mathrm{~d}$ eigenvalues compatible with the Hartree-FockSlater model are not available for plutonium, so that the location and width of the $6 \mathrm{~d}$ bands is unknown. Thus at the present time it is not clear to what extent the interesting TB results can be applied to the two-band model. Subsequent work will involve Herman-Skillman wave functions having configurations with occupied $6 \mathrm{~d}$ levels.

7.4. The APW method leads to a comparatively wider range of energies. The value of about 0.4 Rydberg is comparable to that found for the $3 \mathrm{~d}$ bands in the iron-series transition metals or to the width of the $6 \mathrm{~d}$ band in the cellular calculations Ridley and Lehman did for uranium. The non-relativistic APW results reported here do not escape the problem that if one follows the rules of Fermi-Dirac statistics and fills the lowest available states, then the eight electrons outside the radon core would have predominantly $f$ character and there would he virtilally no electrons that usually would be regarded as conduction electrons. Although suitably narrow bands were found by the IIB calculation, it would appear that only the $5 f$ bands can be readily filled and no conduction or valence electrons would be present unless the prediction of overlapping $6 \mathrm{~d}$ bands is borne out by subsequent calculation. Thus at the present time both calculations will only support the conclusion that eight highly localized $5 f$ electrons would be present.

7.5. Brodsky (31) discusses several band structures in his paper on the Hall coefficient of plutonium. The present non-relativistic calculations 
yield bands which are not in good agreement with any of the three pictures he shows. For example, the spin-orbit splitting in the atom is almost an order of magnitude larger than that suggested by his Figs. $5 b$ and $5 c$ which are derived from the analysis by Rocher. (32) As suggested below, it is not unlikely that the top of the $7 \mathrm{~s}$ band will be in the midst of the $5 \mathrm{f}$ bands as Fig. $5 \mathrm{c}$, which he prefers, indicates. In the authors' opinion, the band structures finally calculated will probably incorporate features from all three figures: One reason why further calculations may eliminate the dissimilarity between the band structure deduced experimentally and that computed is discussed below. 7.6. Fig. 9 indicates that solution of a relativist1c Hamiltonian (Dirac's equation) in the APW calculation will lead to a substantial modification of the bands since the $7 \mathrm{~s}, 6 \mathrm{~d}$ and $5 f$ one-electron eigenvalues are near each other. Further, because of the arrangement of eigenvalues, $7 \mathrm{~s}$ and possibly $6 \mathrm{~d}$-like states probably would be occupied along with $5 f$ states. Consequently, plutonium could be regarded as having a more reasonable number of $s$ and $d$ electrons than can be justified on the basis of the present energy band calculations. A relatiistic APW calculation such as Loucks (33) has recently done for tungsten and lead is planned.

8. Conclusion

8.1. Use of non-relativistic methods of computing the electronic band structure of plutonium has led to interesting but not wholly satisfactory results. The tight binding method involving Herman and Skillman wave functions appropriate for highly localized $5 f$ electrons has yielded narrow bands for both the metal and the monocarbide. For the metal, the band width is in good agreement with that needed by Kmetko to explain the physical properties of metallic plutonium on the basis of his heuristic two-band model. In contrast the APW calculation leads to much broeder bands. The narrowness of the $5 f$ band in PuC, indicated by the tight binding method, implies a possibly large electronic specific heat at room temperature and above, of the order of several calories per mole. 
1. J. A. Lee and R. O. A. Ha]l, J. Less-Camm. Metals 1959, 1, 356. See also M. B. Waldron, et al, "Proceedings of the 2nd International Conference on Peaceful Uses of Atamic Energy", Vol. 6, p. 1621958 Geneva (United Nations)

2. E. C. Ridley, Proc. Roy. Soc. (London), 1958, A247, 199

3. D. J. Howarth, Proc.. Roy. Soc. (Iondon), 1958, A220, 513

4. G. W. Lehman, Phys. Rev. 1960, 117, 1493

5. J. C. Slater, Phys. Rev. 1937, 51, 846

6. N. F. Mott and H. Jones, "Theory of the Properties of Metals and Alloys", p. 65, 1963 Oxford (Clarendon Press)

7. E. Kmetko, to be published

8. P. Costa and R. R. Conte, Int. Symp. "Compounds of Interest in Nuclear Reactor Technology", (Boulder, 1964) p.3 1964 New York (Amer. Inst. Min. Met. Eng.)

9. V. Ern and A. C. Switendick, Phys. Rev. 1965, 137, Alg27

10. R. E. Rundie, Acta Cryst. 1948, I, 180

11. F. Herman and S. Skillman, "Atomic Structure Calculation", p. 6-21l, 1963 tinglewood Cliffs (Prentice-Hall)

12. E. P. Wigner and F. Seitz, Phys. Rev. 1933, 43, 804

13. J. E. Barnes, to be published

14. N. F. Mott and H. Jones, op. cit. p. 67

15. L. P. Bouckaert, R. Smoluchowski and E. Wigner, Phys. Rev. 1936, 50, 58

16. J. H. Woor, Phys. Rev, 1962, 126, 517

17. L. H. Mattheiss, Phys. Rev. 1964, 133, Al399

18. P. 0. Löwdin, Advances Phys. 1956, 2, I

19. J. C. Slater, Phys. Rev., 1951, 81, 385

20. G. A. Burdick, Phys. Rev. 1963, 129, 138

21. J. T. Waber and A. C. Switendick, submitted to 5th Rare Earth Conference, Ames, Iowa, 1965

22. E. C. Snow, J. T. Waber and A. C. Switendick, submitted to 1 th Magnetism and Magnetic Materials Conference, San Francisco, California, 1965

23. A. C. Larson and J. T. Waber, "Proc. 3rt Rare Earth Conf. (Clearwater, I.963)" p. 3511964 New York (Gordon and Breach) 
24. J. T. Waber, D. Liberman and D. T."Cromer, "Proc. 4th Rare Earth Conf. (Phoenix 1964)", in press, New York (Gordon and Breach)

25: R. G. Boyd; A. C. Larson and J. T. Waber, to be published

26. D. Liberman, J. T. Waber and D. T. Cromer, Phys. Rev. 1965, 137, A27

27. R. G. Boyd, A. C. Iarson and J. T. Waber, Phys. Rev. 1963, 129, 1629

28. L. Weil, G. Quezel, J. Cohen and R. Pascard, "Plutonium 1960" p. 104, 1961 London, (Cleaver-Hume Ltd.). See also Lunsford and.Kmetko, this Conference

29. E. C. Snow, J. M. Canfield and J. T. Waber, Phys. Rev. 1964, 135, A969

30. W. H. Zachariasen, "The Metal Plutonium", p. 99, 1961 Chicago (Univ. of Chicago Press)

31. M. Brodsky, Proc. this Conference

32. Y. A. Rocher, Phil. Mag. Suppl. 1962 II No. 43, 233

33. T. L. Loucks, Phys.' Rev. Lettr. 1965, 14, 693, also 1072 
Table I

Comparison of the One-Electron Eigenvalues for the Free Plutionium Atom

\begin{tabular}{|c|c|c|c|c|c|c|c|}
\hline \multirow{2}{*}{$\begin{array}{l}\text { Authors' } \\
\text { Model } \\
\text { nlj }\end{array}$} & \multicolumn{3}{|c|}{$\begin{array}{c}\text { Boyd-Larson-Waber } \\
\text { (Hartree) }\end{array}$} & \multirow{2}{*}{$\frac{\begin{array}{c}\text { Herman-Skillman } \\
\text { (Hartree-Fock-Slater) }\end{array}}{f^{6}}$} & \multicolumn{3}{|c|}{$\begin{array}{l}\text { Liberman-Waber-Cromer } \\
\text { (Dirac-Slater) }\end{array}$} \\
\hline & $x^{6}$ & $\mathrm{f}^{5} \mathrm{~d}^{1}$ & $i^{4} a^{2}$ & & $\mathbf{1}^{6}$ & $\mathrm{f}^{5} \mathrm{~d}^{1}$ & $f^{4} d^{2}$ \\
\hline $5 f^{\frac{5}{2}}$ & 0.571 & 0.885 & 1.192 & 1.073 & 0.505 & 0.783 & 1.063 \\
\hline $6 s \frac{1}{2}$ & 2.602 & 2.826 & 3.024 & 2.903 & 3.894 & 4.135 & 4.366 \\
\hline $6 p \frac{1}{2}$ & & & & $70-7$ & 2.476 & 2.687 & 2.887 \\
\hline $6 \mathrm{p} \frac{3}{2}$ & 1.559 & 1.715 & 1.915 & 1.831 & 1.703 & 1.875 & 2.0 .35 \\
\hline $6 \mathrm{~d} \frac{3}{2}$ & -- & 0.301 & 0.375 & -- & - & 0.330 & 0.404 \\
\hline $7 s \frac{1}{2}$ & 0.297 & 0.319 & 0.330 & 0.336 & 0.393 & 0.430 & 0.460 \\
\hline
\end{tabular}

The negative sign of these eigenvalues has been dropped for convenience. For the purpose of this table the number of $7 \mathrm{~s}$ electrons is two. 


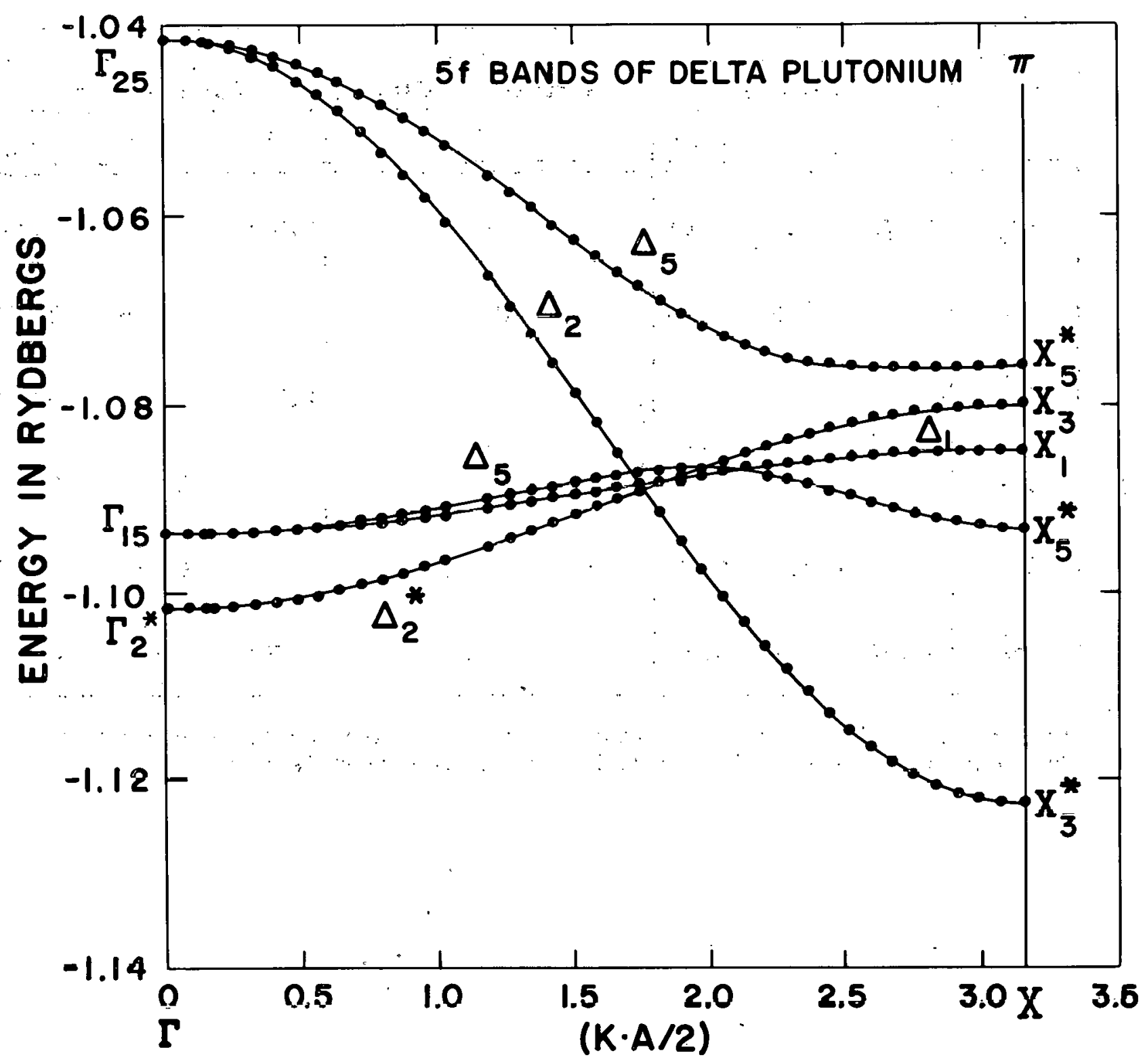

Fig. 1 The energy bands or $\mathrm{E}(\mathrm{k})$ curves for the $5 f$ states in delta plutonium, drawn for the high symmetry direction $r \Delta X$ or $(k, 0,0)$. The abscissa is a dimensionless distance involving the wave vector $k$ and the lattice constant a. Notation of states follows that of Bouckaert, Smoluchowski and Wigner. 


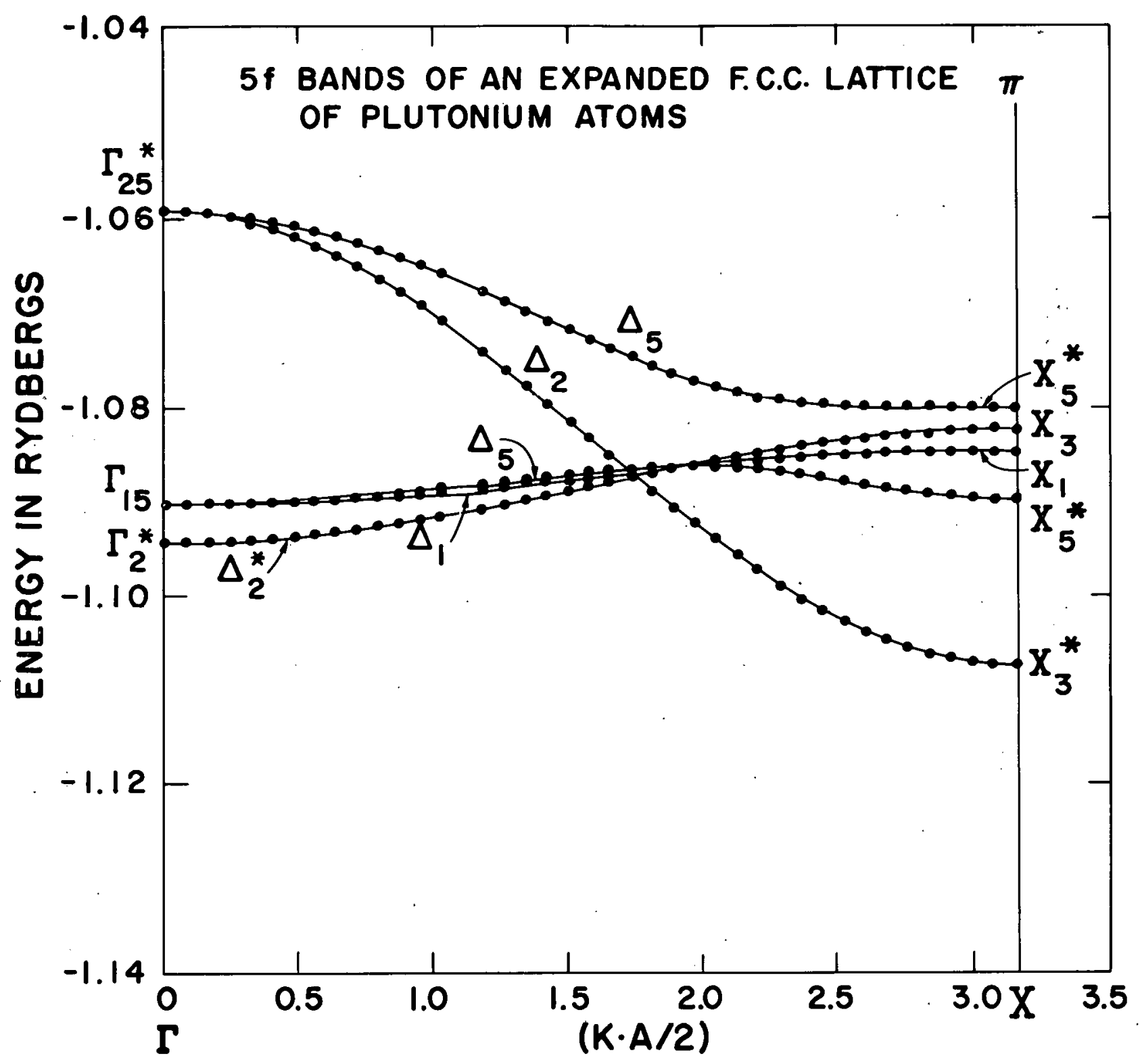

Fig. 2 The $5 f$ bands for the hypothetical F.C.C. lattice of plutonium atoms expanded to the lattice parameter $1.07 \mathrm{a}$. This is the metal sublattice in PuC. 


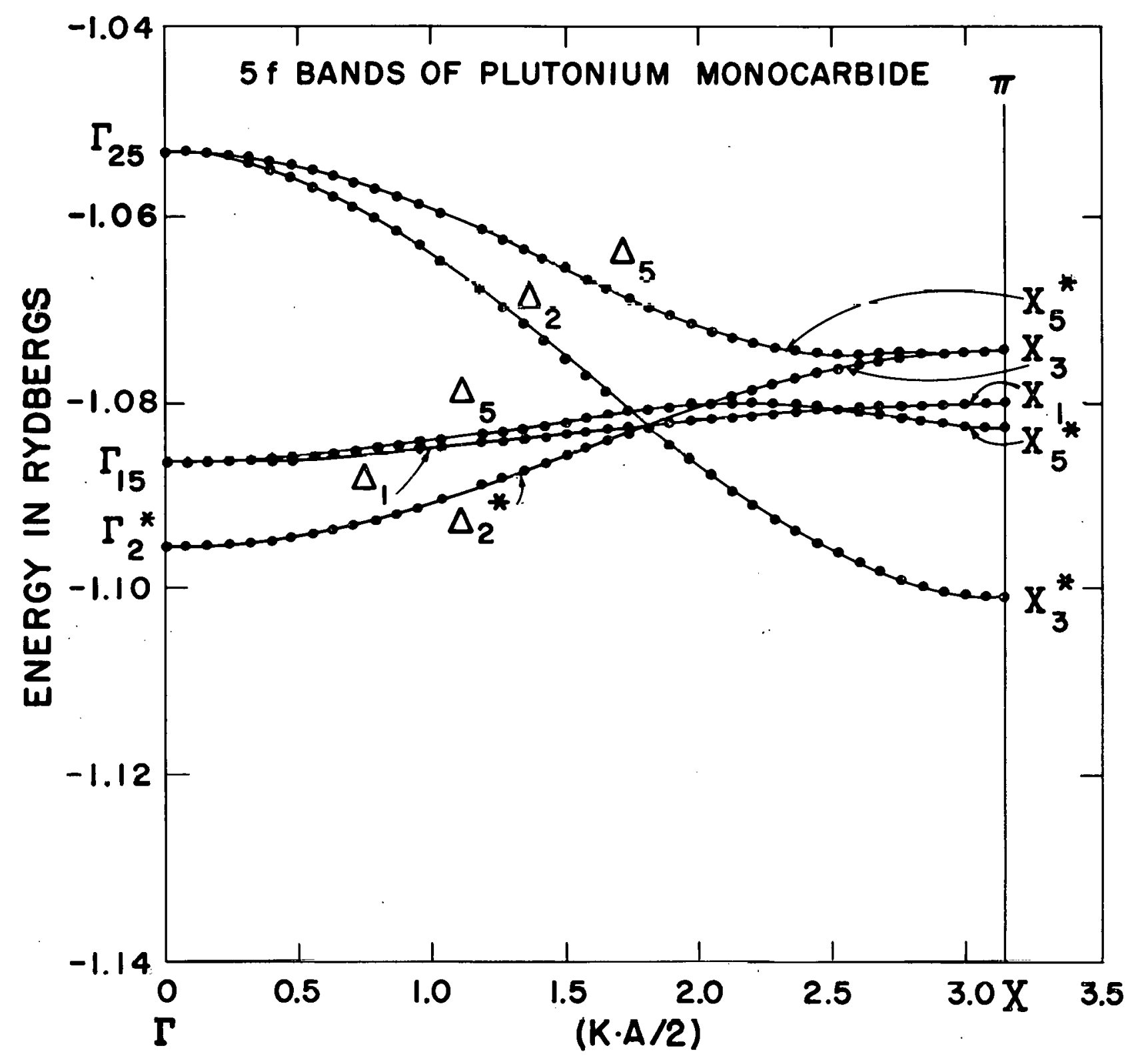

Fig. 3 The $5 f$ bands for PuC, which crystallizes in the NaCl structure. 
CONDUCTION AND ATOMIC CORE BANDS OF DELTA PLUTONIUM

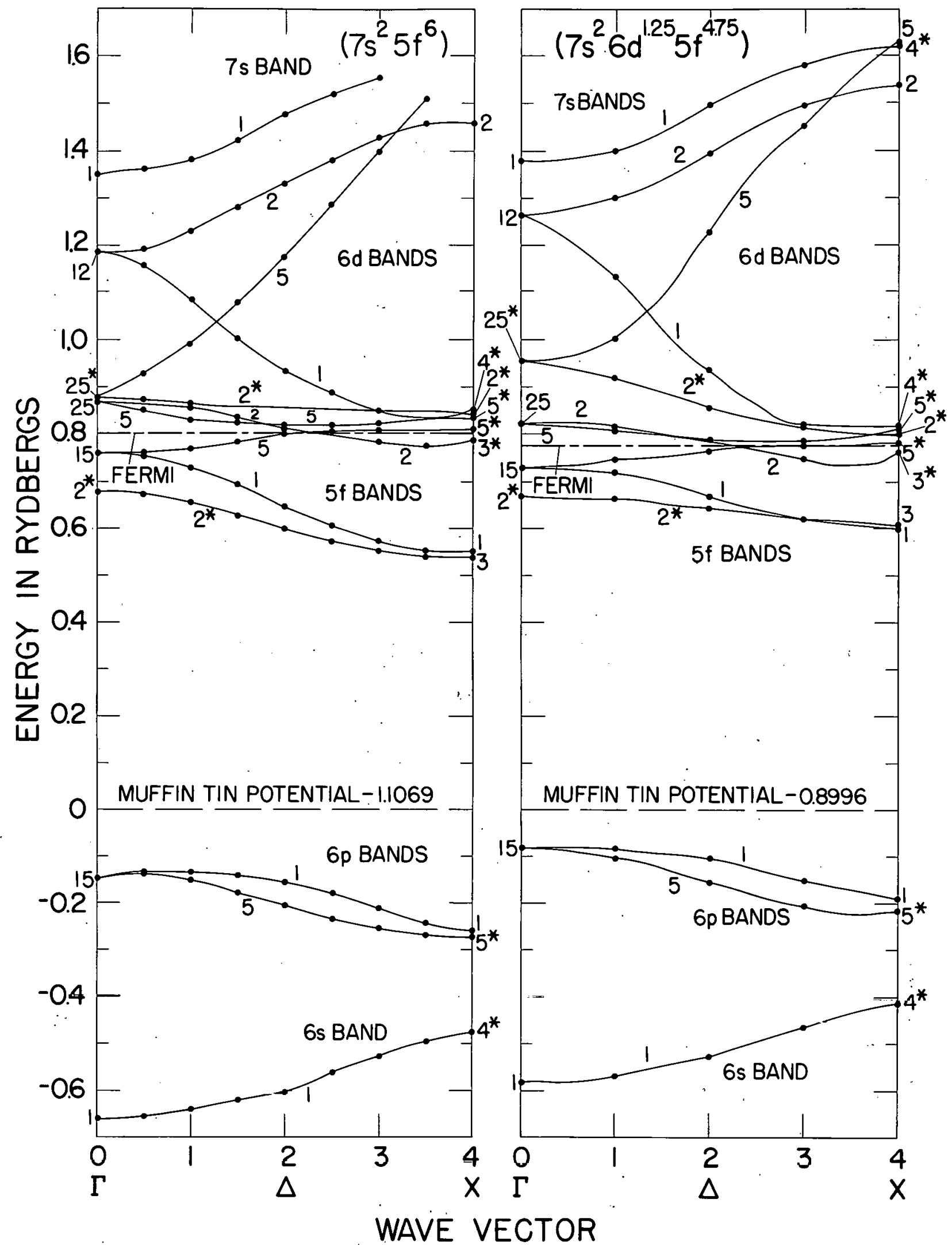

Fig. 4 A comparison of the band structure for two assumed configurations of plutonium atoms. The dominant nature of the various conduction and core bands is indicated. The abscissae are the wave vectors $(k, 0,0)$ in units of $(\pi / a)$. Because of the greater number of bands, only the subscripts are used as labels. For example, the state $r_{2}^{*}$ is labeled as $2^{*}$. 
ENERGY' IN RYDBERGS'

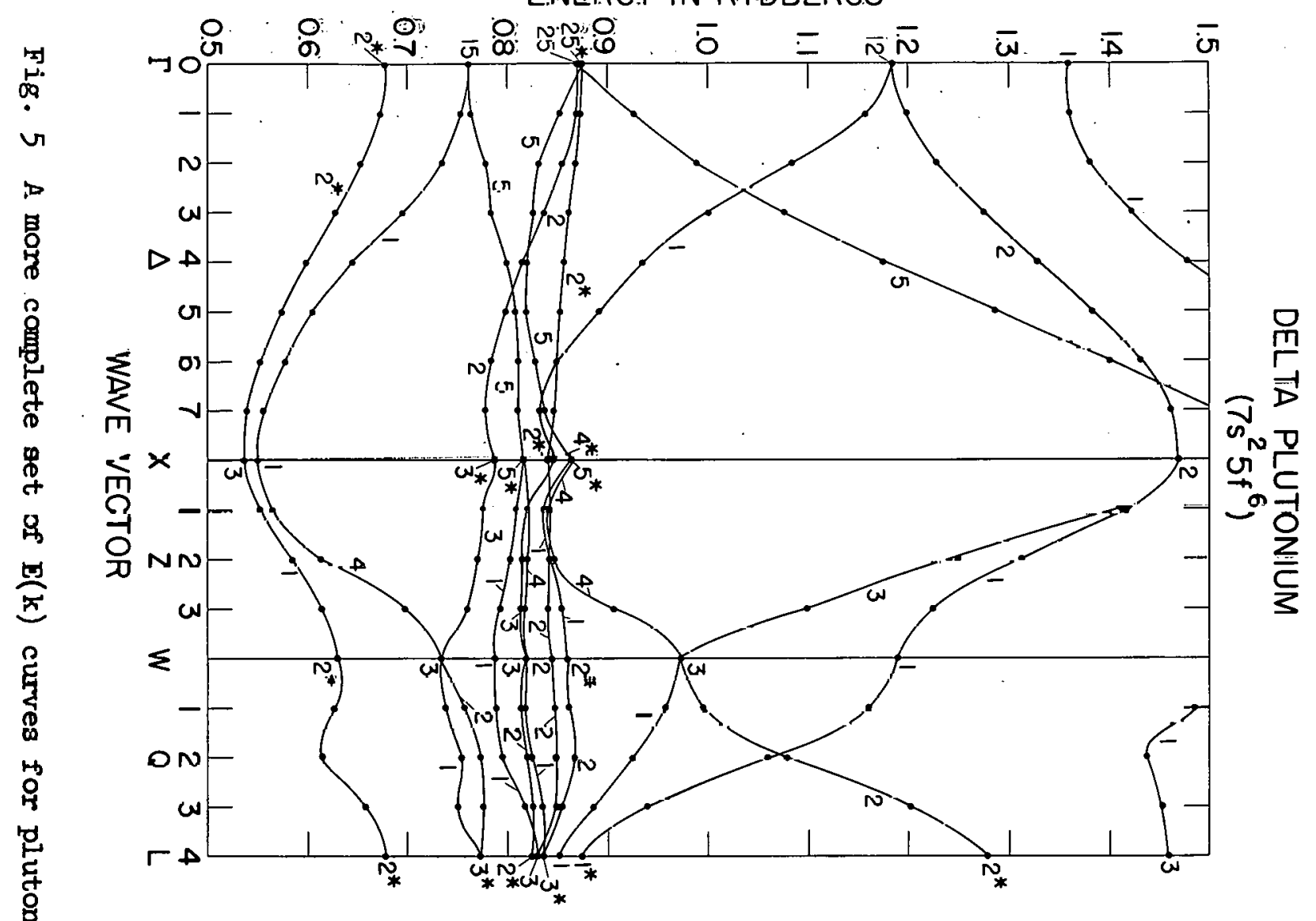

ENERGY IN RYDBERGS

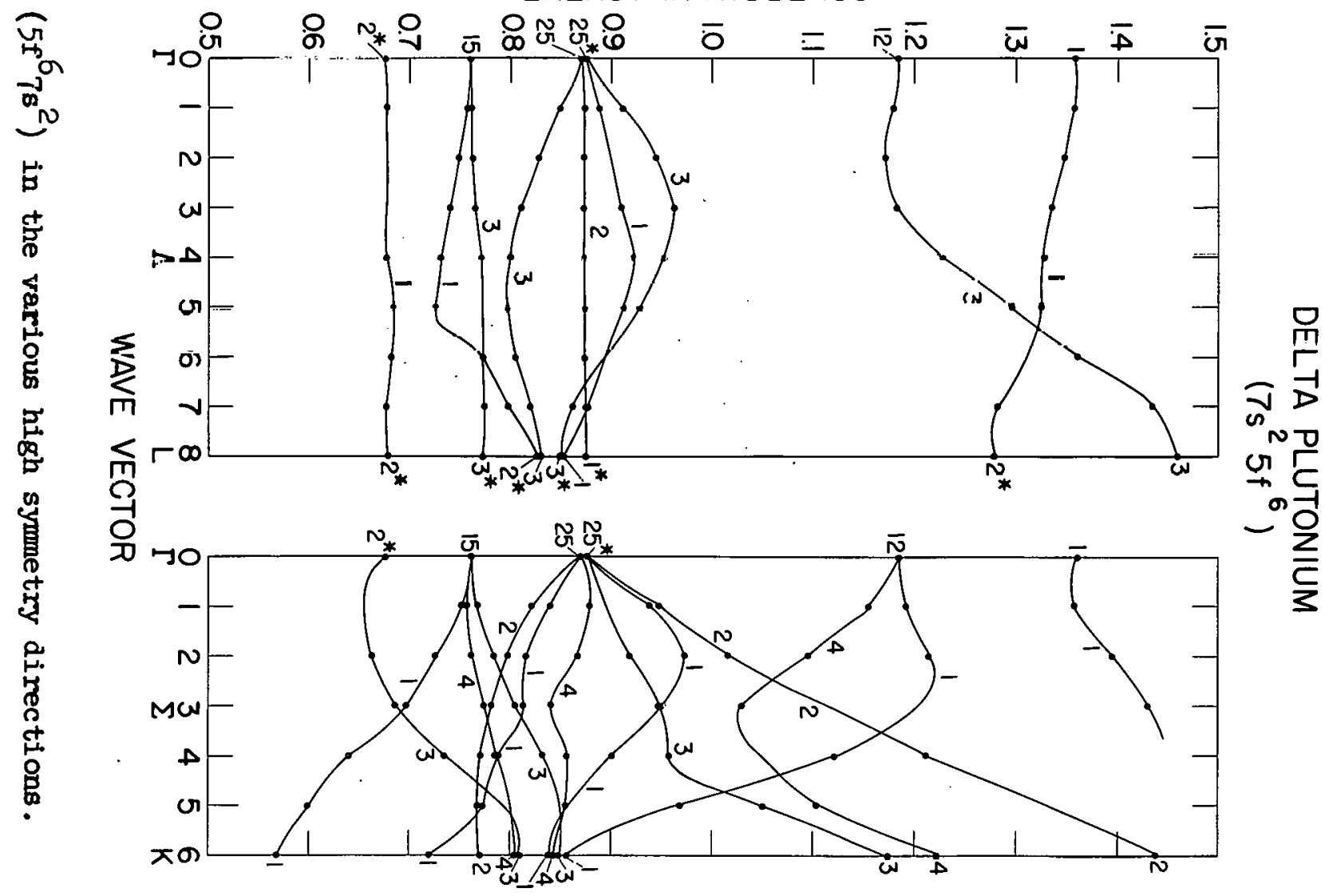




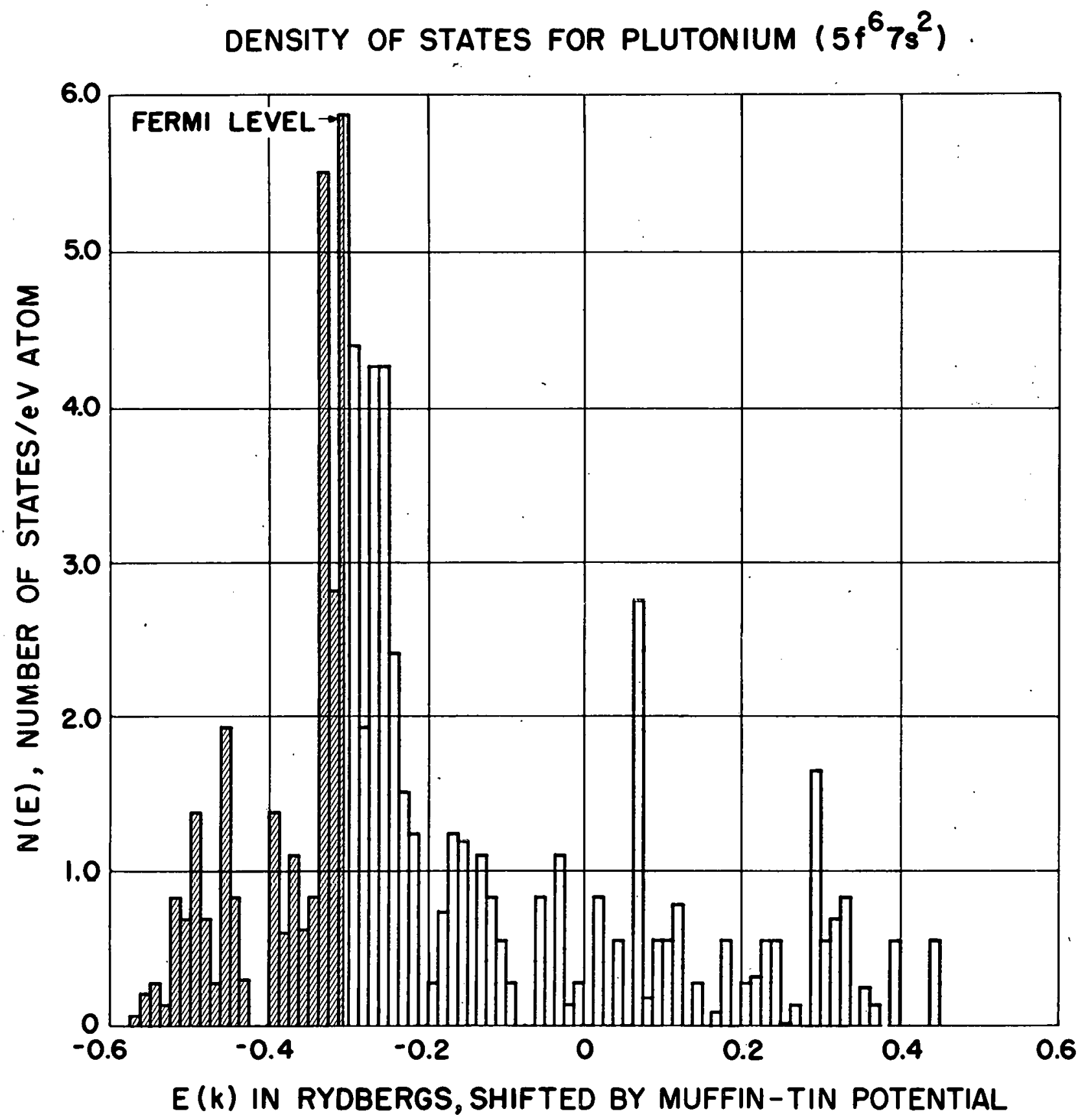

Fig. 6 Density of states histogram for the Augmented Plane Wave calculation of plutonium $\left(5 f^{6} 7 s^{2}\right)$. The indicated Energy or "Fermi" level accommodates elght electrons. 


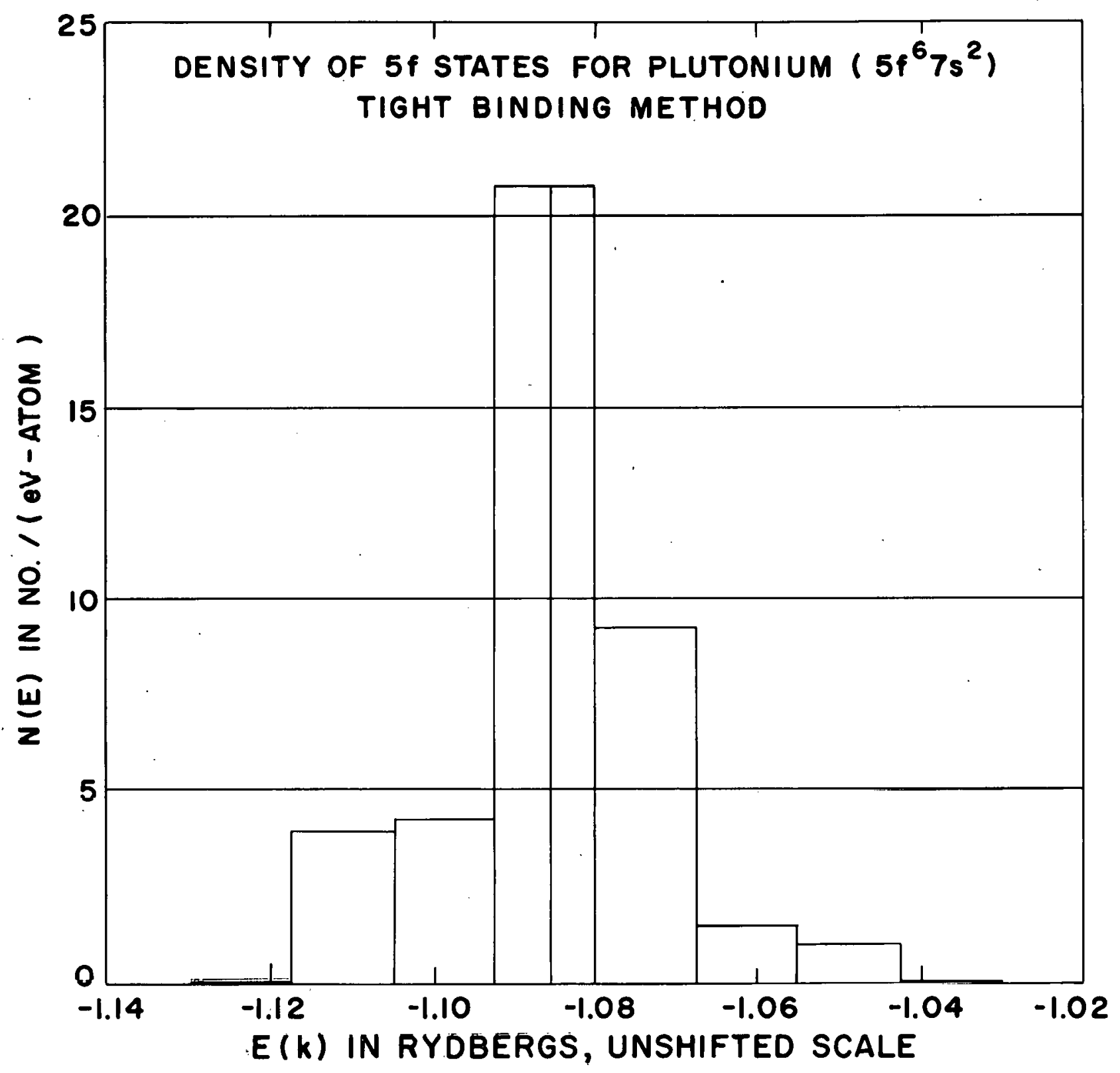

Fig. 7 Density of states histogram for the tight binding calculation of plutonium $\left(5 \mathrm{f}^{6} 7 \mathrm{~s}^{2}\right)$. 


\section{SECTIONS OF THE BRILLOUIN ZONE FOR A FCC METAL}

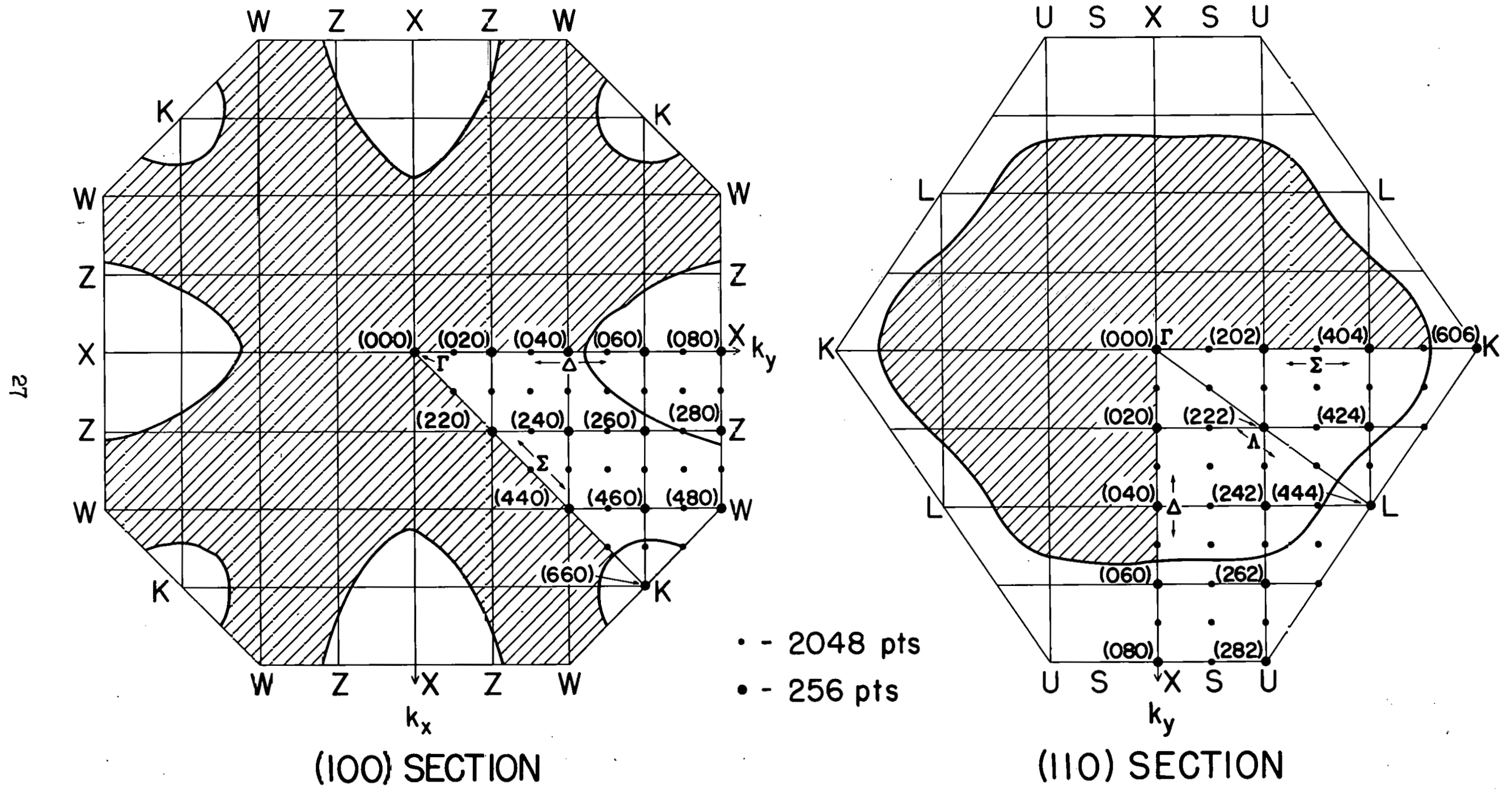

Fig. 8 Two sections through the Brillowin zone $i$ lustrating the shape of the energy contour which corresponds to the fourth band. 
EFFECT OF CONFIGURATION ON THE ONE - ELECTRON EIGENVALUES OF A CONFINED PLUTONIUM ATOM.

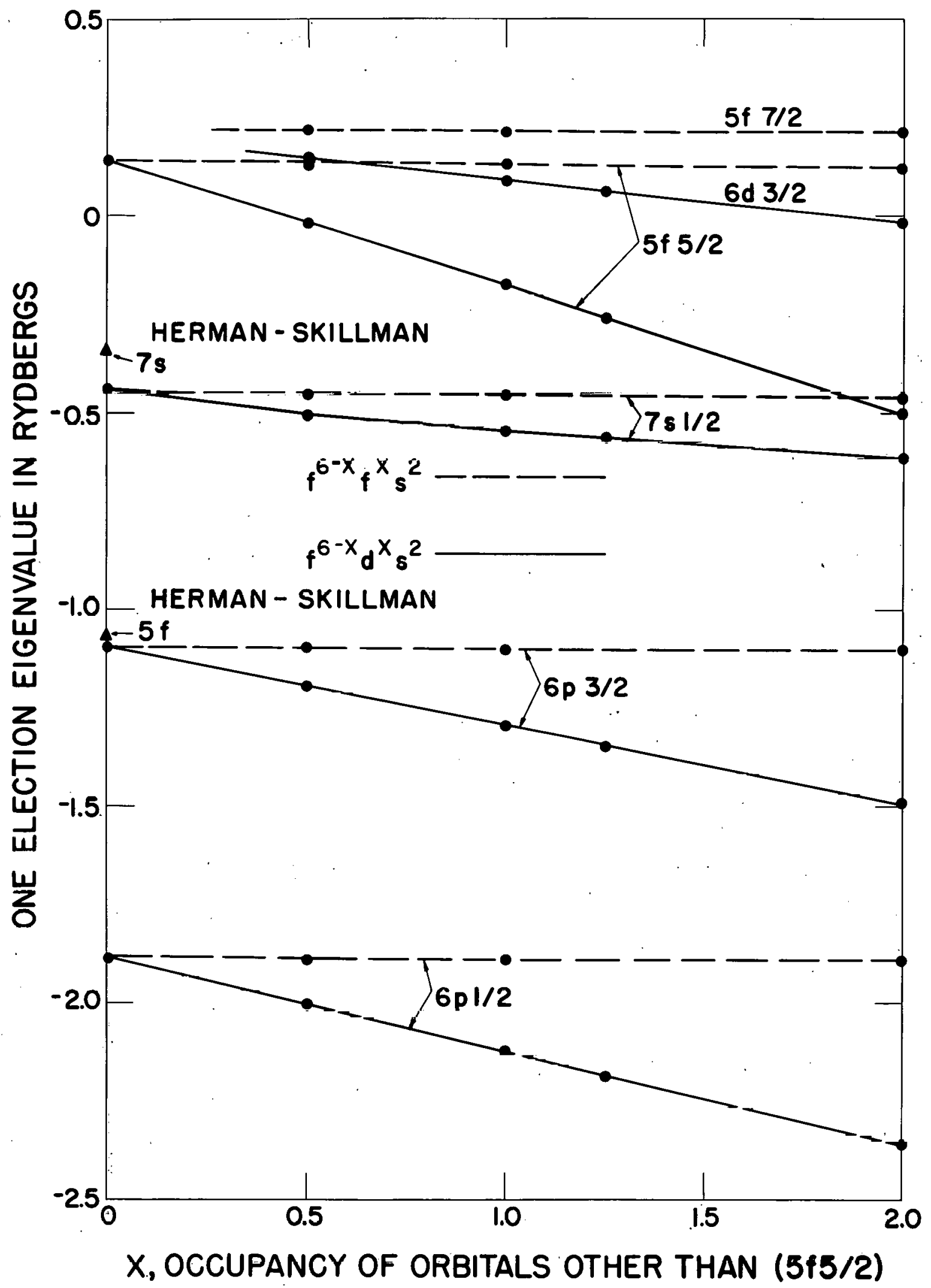

Fig. 9 The effect of assuned configuration on the one-electron energy eigenvelues obtained with Dirac-Slater wave functions for a plutonium atom confined to a Wigner-Seitz sphere of radius; 3.46 Bohr units. The free atom eigenvaliues of Herman and Skililman are also shown. 


\section{VARIATION OF THE TOTAL ENERGY OF A CONFINED PLUTONIUM ATOM WITH CONFIGURATION.}

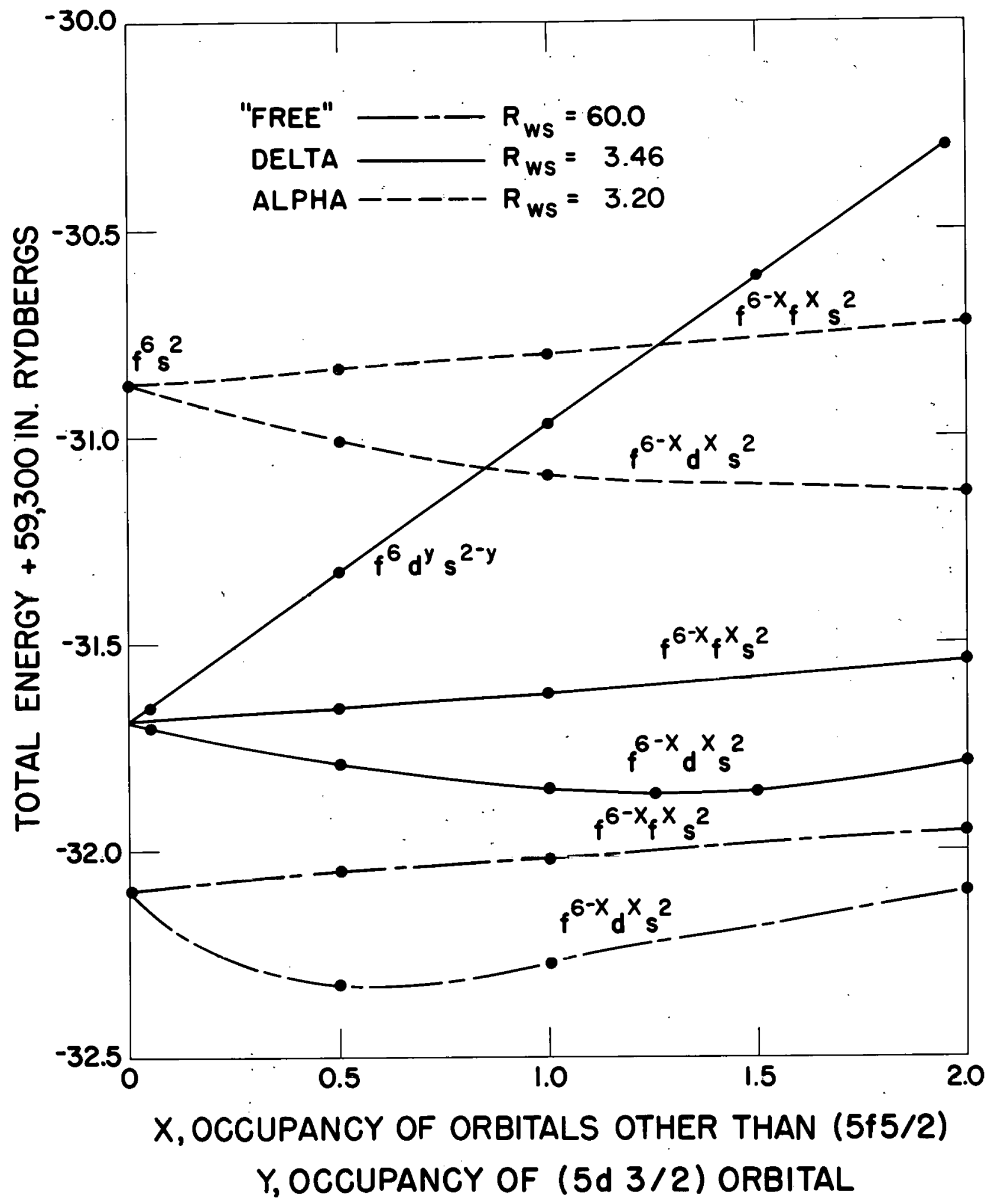

Fig. 10 Effect of configuration for three radii on the total energy $E_{T}$ of a
confined plutonium atom. 


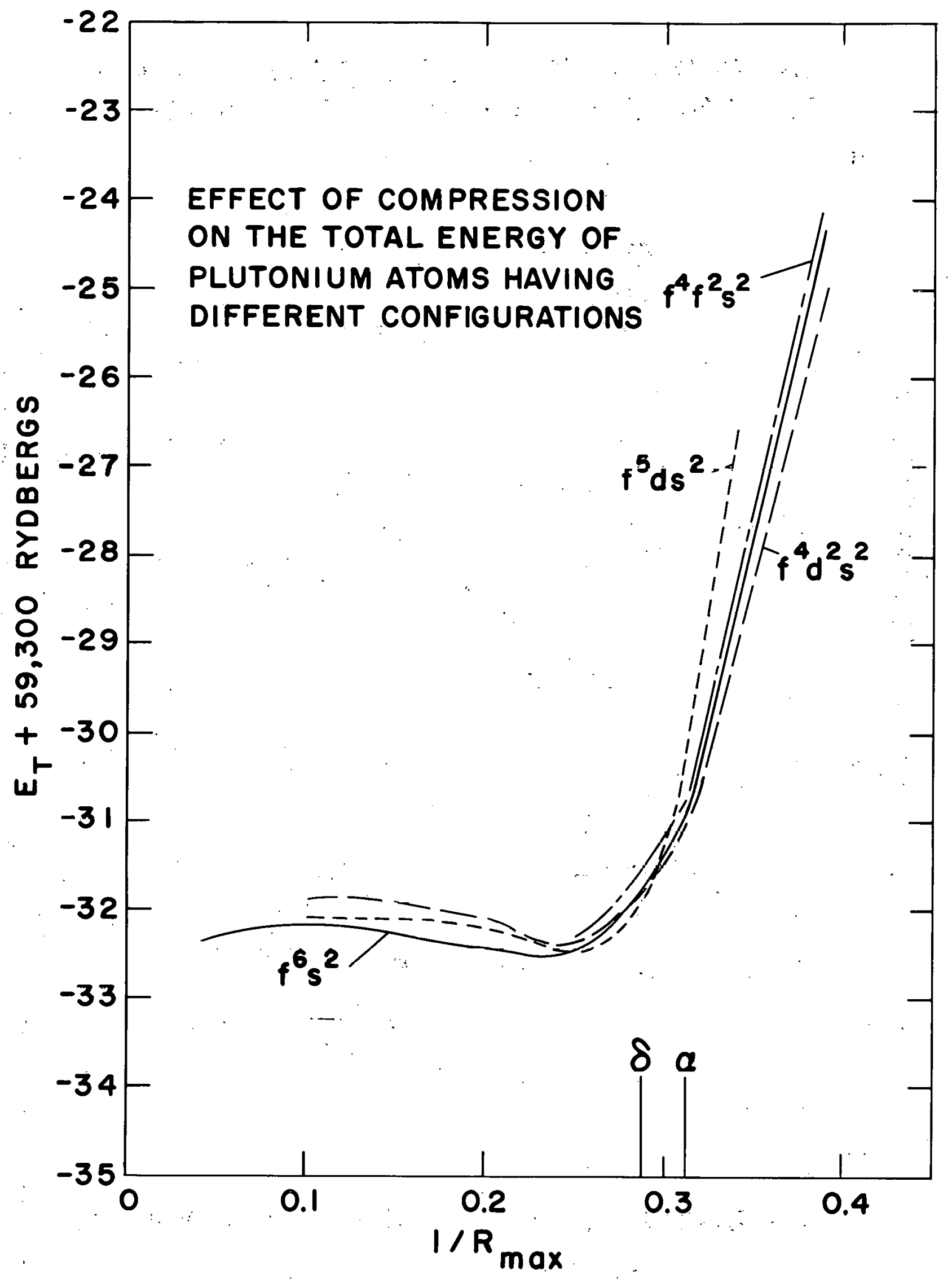

Fig. Il Effect of radius for four configurations on the total energy of a confined plutonium atom. 


\section{LIST OF FIGURES}

Fig. I The energy bands or $E(k)$ curves for the $5 f$ states in delta plutonium, drawn for the high symetry direction $r \Delta X$ or $(k, 0,0)$. The abscissa is a dimensionless distance involving the wave vector $k$ and the lattice constant a. Notation of states follows that of Bouckaert, Smoluchowski and Wigner.

Fig. 2 The $5 f$ bands for the hypothetical F.C.C. lattice of plutonium atoms expanded to the lattice parameter $1.07 \mathrm{a}$. This is the metal sublattice in Puc.

Fig. 3 The $5 f$ bands for PuC, which crystallizes in the NaCl structure.

Fig. 4 A comparison of the band structure for two assumed configurations of plutonium atoms. The dominant nature of the various conduction and core bands is indicated. The abscissae are the wave vectors $(k, 0,0)$ in units of $(\pi / a)$. Because of the greater number of bands, only the subscripts are used as labels. For example, the state $\mathbf{r}_{2}^{\prime}$ is labeled as $2^{*}$.

Fig. 5 A more complete set of $E(k)$ curves for plutonium $\left(5 f^{6} 7 s^{2}\right)$ in the various high symetry directions.

Fig. 6 Density of states histogram for the Augmented Plane Wave calculation of plutonium $\left(5 f^{6} 7 s^{2}\right)$. The indicated Energy or "Fermi" level accommodates eight electrons.

Fig. 7 Density of states histogram for the tight binding calculation of plutonium $\left(5 \mathrm{f}^{6} 7 \mathrm{~s}^{2}\right)$.

Fig. 8 Two sections through the Brillouin zone illustrating the shape of the Fermi surface. Energy contour corresponds to the fourth band.

Fig. 9. The effect of assumed configuration on the one-electron energy eigenvalues obtained with Dirac-Slater wave functions for a plutonium atom confined to a Wigner-Seitz sphere of radius 3.46 Bohr units. The free atom eigenvalues of Herman and Skillman are also shown.

Fig. 10 Effect of configuration for three radii on the total energy $E_{T}$ of a confined plutonium atom.

Fig.1l Effect of radius for four configurations on the total energy of a confined plutonium atom. 